\title{
The LHC Pre-Series Dipole Cold Mass Geometry
}

\author{
M. Bajko, R. Chamizo, F. Savary, B. Skoczen, R. Veness, and J. B. Jeanneret
}

\begin{abstract}
In order to provide the necessary mechanical aperture for the LHC beam, the main dipole cold masses have to match precisely the nominal circular trajectory of the particles beam. The requirements on the dipole cold mass geometry are dictated by the LHC beam optics and by the allowed limits of mechanical deformation of the interconnection bellows. Keeping the tight tolerances that are imposed necessitates a well controlled bending process and the use of a high accuracy 3D measuring instrument for checking the geometry of the cold mass throughout many manufacturing stages up to the final inspection.

The dipole cold mass pre-series production started in 2000. It is almost completed at the three sites. In this paper, we report on the problems encountered to shape correctly the cold masses, their effect on interconnection of the dipole cold masses and on the mechanical aperture. On one side measures to improve the production process in terms of accuracy and reproducibility were taken, on the other side the assembly tolerances could be relaxed following a thorough review of the machine requirements.

The summary of the encountered problems, corrective actions and results obtained on the pre-series cold masses are presented and discussed.
\end{abstract}

Index Terms-Alignment, cold mass, dipole, geometry, LHC.

\section{INTRODUCTION}

$\mathbf{T}$ HE LHC dipole cold mass pre-series production (30 units by firm and about $7 \%$ of the whole production) started in 2000 and is today about completed. During this starting phase, two general reviews were held at CERN, to evaluate the feasibility of the dipole cold mass production in industry. In particular, the following issues were analyzed and discussed: the geometry of the cold masses, the measurement guided assembly procedure and the feasibility of the required mechanical tolerances. Based on the outcomes of the first review, the mechanical tolerances on the extremities of the cold masses were relaxed. The change has been agreed thanks to an optimization work, between the magnet designers and the responsible of the interconnections of the magnets in the LHC tunnel.

Analysis made for the preparation of the second review, highlighted the difficulties to obtain the specified mechanical aperture. Problems arise from the bending process of the dipole cold masses and geometrical instabilities. In view of solving these problems, two solutions were proposed without making changes of the dipole cold mass structure: an optimization of the tolerances of the mechanical aperture or/and the alignment of the magnets by the ends.

Manuscript received October 20, 2003.

The authors are with the CERN, Geneva, CH (e-mail: Marta.Bajko@ cern.ch; Rocio.Chamizo@cern.ch; Frederic.Savary@cern.ch; Blazej.Skoczen@cern.ch; Ray.Veness@cern.ch; Bernard.Jeanneret@cern.ch).

Digital Object Identifier 10.1109/TASC.2004.829050

\section{REFERENTIAL FOR THE POSITIONING DURING THE ASSEMBLY AND ALIGNMENT IN THE LHC TUNNEL}

The dipole cold masses for the LHC are produced in three firms in Europe. After the delivery of them to CERN they are cryostated, tested and stored, waiting for transport and alignment in the tunnel. Each dipole cold mass is about $15 \mathrm{~m}$ long and $27 \mathrm{t}$ heavy. It is supported in three points in such a way that there is not any constraint in its horizontal plane. The flexural rigidity of a cold mass has been evaluated to be approximately equivalent to the rigidity of their shrinking cylinders, which are made of AISI 316LN stainless steel, with an outer diameter of $550 \mathrm{~mm}$ and $10.5 \mathrm{~mm}$ thickness [1]. A reference, fixed on the dipole cold mass would move along the assembly phases, depending on the supporting and other external conditions. In order to have a stable and common reference for the dipoles, the beam trajectory, a theoretical curve, was chosen as referential.

\section{The GeOMETRY OF THE LHC Dipole Cold MASSES AND THE BASE LINE TOLERANCES}

For the requirements of the geometry of the cryo-dipoles there are two types of considerations: beam optics and mechanical. For the beam optics, the largest mechanical aperture is the main requirement. The imperfections of the dipole field are corrected with multipolar magnets, which are integral parts of the dipole cold mass and therefore, their position is strictly linked to the geometry of the dipole. The second consideration is mechanical and is linked to the interconnections of the magnets in the LHC tunnel by flexible bellows.

\section{A. The Shape of the Dipole Cold Mass}

The trajectory of the beam over the length of one dipole is considered in the horizontal plane as composed by a portion of circle with a radius of $2812.36 \mathrm{~m}$ corresponding to an apical angle of $5.09 \mathrm{mrad}$ and two straight lines, tangent to the arc. The length of the bent part is $14343 \mathrm{~mm}$ in assembly conditions. This theoretical geometric axis is identical for the second aperture of the twin dipoles and the center of its bent part is $194.52 \mathrm{~mm}$ apart at room temperature. In the vertical plane the geometrical axes are straight lines.

\section{B. The Mechanical Tolerances on the Shape and on the Position of the Different Components}

The overall budget of errors for the mechanical aperture, the position of the corrector magnets and the different components of the cold mass to be interconnected in the LHC tunnel, is shared between the different phases of the assembly and installation of the dipoles in the tunnel. 


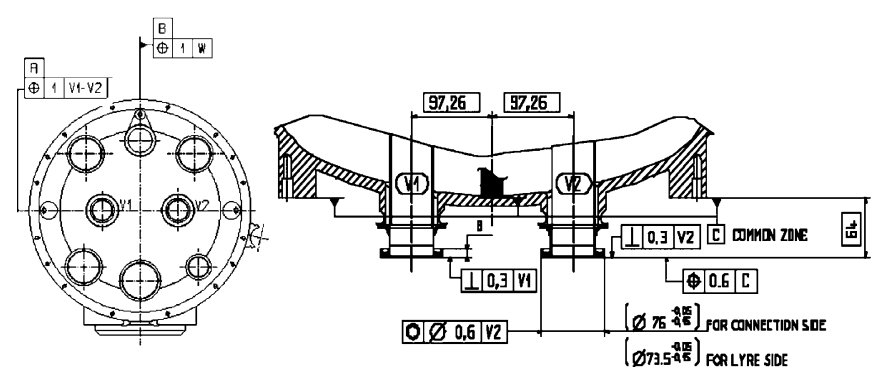

Fig. 1. The theoretical geometrical axes: V1 and V2. The tolerances on the position of the end cover and on the cold bore tube ends.

1) Tolerance on the Shape: The global tolerance range on the shape is $2 \mathrm{~mm}$, from which, for the assembly itself is allocated $1 \mathrm{~mm}$. This represents the acceptable radial deviation between the theoretical geometrical axes and the axes of the cold bore tubes. On the manufacturing drawings this is given as a set of two toroidal sectors of circular section and 4 straight cylinders, with a radius of $1 \mathrm{~mm}$, centered on the geometrical axes.

2) Tolerance on the Interconnecting Components: The extreme limit of the interconnecting bellows is $2 \mathrm{~mm}$. Taking into account the different steps of assembly of the cryo-dipole, the allowable tolerance on the cold bore tube extremity for the manufacturing is $0.3 \mathrm{~mm}$ radially. On the end covers, there are three additional nozzles, equipped with bellows to be interconnected; therefore the positioning of the end cover is limited to $\pm 0.5 \mathrm{~mm}$ with respect to its nominal position. To reduce this error, the end covers are also aligned with respect to the theoretical geometrical axes (See Fig. 1).

The overall length of the cold mass is $15158 \mathrm{~mm} \pm 2 \mathrm{~mm}$. To respect the tolerance of the shape, in the vertical plane, the cold feet pads have to be positioned with respect to their nominal position, of $5400 \mathrm{~mm}$ inter-distance, within a tolerance of $\pm 1 \mathrm{~mm}$. The plane of the feet should stay at the same height: $294 \mathrm{~mm}$ from the plane containing the theoretical geometrical axes, within a tolerance of $\pm 0.5 \mathrm{~mm}$.

3) Tolerance on the Corrector Magnets: For the position of the corrector magnets (sextupoles: MCS and combined decapole octupole magnets: $\mathrm{MCD} / \mathrm{MO}$ ), the beam dynamics requires, a position with a maximum of $\pm 0.3 \mathrm{~mm}$ systematic, and of $0.5 \mathrm{~mm}$ random deviation $(1 \sigma)$ from the geometrical axes of the dipoles. This requirement is shared between the manufacturing of the corrector magnets, the assembly and installation of the correctors in the dipoles, and finally the installation of the cryo-dipoles. The manufacturing tolerances of the correctors are $0.1 \mathrm{~mm}$ mean error and $0.1 \mathrm{~mm}$ random error at $1 \sigma$.

\section{REVIEW OF THE TOLERANCES ON THE EXTREMITY OF THE LHC DIPOLE COLD MASSES}

Following great difficulties reported by the manufacturers, a review of the tolerances was necessary. The imposed tolerances for the positioning were unrealistic because their achievement necessitates too much time.

A statistical analysis was made to study the industrial feasibility of the required tolerance. The study was based on the results obtained on the first 20 magnets. In spite of the fact that the measurements show that the components are within the
TABLE I

SuMmaRy OF Results ObTAINED ON THE FIRST 20 MAgNETS

\begin{tabular}{lcccc}
\hline \hline \multicolumn{1}{c}{ Parameter } & $\begin{array}{c}\text { Normal } \\
\text { value }\end{array}$ & $\begin{array}{c}1 / 2 \text { of the } \\
\text { tolerance range }\end{array}$ & Mean & $3 \sigma$ \\
\hline Length & 15158 & 2 & 15158 & 2.83 \\
End cover position $(\mathrm{mm})$ & 0 & 0.5 & 0.09 & 0.63 \\
End cover angle $(\mathrm{mrad})$ & 0 & 0.4 & 0.04 & 0.83 \\
End flange position $(\mathrm{mm})$ & 0 & 0.3 & 0.15 & 0.59 \\
End flange angle $(\mathrm{mrad})$ & 0 & 4 & 2.77 & 7.22 \\
End tube length $(\mathrm{mm})$ & 64 & 0.3 & 64.06 & 1.2 \\
\hline \hline
\end{tabular}

TABLE II

BASE LiNE AND REviewed TOLERANCES. Status OF THE PRODUCTION IN OCTOBER 2003

\begin{tabular}{|c|c|c|c|c|c|}
\hline \multirow[t]{2}{*}{ Parameter } & \multirow{2}{*}{$\begin{array}{l}\text { Normal } \\
\text { value }\end{array}$} & \multicolumn{2}{|c|}{$\begin{array}{c}1 / 2 \text { of the } \\
\text { tolerance range }\end{array}$} & \multirow[t]{2}{*}{ Mean } & \multirow[t]{2}{*}{$3 \sigma$} \\
\hline & & Base line & reviewed & & \\
\hline Length (mm) & 15158 & 2 & 2.2 & 15158.58 & 3.66 \\
\hline End cover position (mm) & & 0.5 & 0.75 & 0.05 & 0.77 \\
\hline End cover angle (mrad) & & 0.4 & 1 & 0.03 & 0.55 \\
\hline End flange position (mm) & & 0.3 & 0.6 & 0.14 & 0.26 \\
\hline End flange angle (mrad) & & 4 & 5 & 1.88 & 4.16 \\
\hline End tube length (mm) & 64 & 0.3 & 0.5 & 64.01 & 0.33 \\
\hline
\end{tabular}

tolerance, the important spread (the standard deviation at $3 \sigma$, comparable with the tolerance) indicates that the tolerances required, are difficult to keep in an industrial process and especially during the learning phase.

The results on the first 20 magnets, showed a large spread in comparison with the expected standard deviation.

The positioning operations were found time consuming, not expected by the companies. For a large-scale production this was not acceptable, therefore the mechanical tolerances were reviewed and relaxed in such a way that the specification for the correctors was not modified.

Relaxing on the tolerances has necessitated an optimization of the interconnecting parameters. In particular a revision of the set-up points for the bellows expansion joints, verification of the effect of combined longitudinal and transverse offsets on the bellows reliability and introduction of a supplementary mechanism of compensation by angular rotation of the interconnection sleeves were necessary. Typically, some of the bellows expansion joints both in the beam lines and in the cryogenic interconnections can loose up to $50 \%$ of their fatigue life as a result of a combined offset.

Another severe limitation taken into account in the process of revision of the tolerances was based on the assembly procedures of the LHC interconnections. The assembly of the electrical interconnections (splices of the superconducting bus-bars) and of the vacuum and the cryogenic lines were reviewed. The new set of relaxed tolerances that satisfy the above-specified limitations is shown in Tables I and II. A summary of the results obtained on the production of the first 80 magnets is presented also.

The relaxed tolerances lead to an important gain in time, especially during the learning phase of the cold mass production.

\section{REVIEW OF THE REQUIREMENTS ON THE TWIST OF THE DIPOLE COLD MASSES}

The local twist of the dipole cold mass is defined as the angle between the line in the transversal section containing the centers 
of the two cold bore tubes in a cold mass and the cold mass horizontal plane. The local twist is limited to $\pm 3 \mathrm{mrad}$ and the average tilt is restricted to $\pm 1 \mathrm{mrad}$.

Although this definition is linked to the mechanical geometry of the cold mass, it is assumed that the geometry of the cold bores represents the geometry of the collared coils and therefore, the direction of the magnetic field along the dipole cold mass. The twist is limited in order to restrict the vertical misalignment of the two coils that would induce a horizontal component of the magnetic field and therefore a vertical deflection of the beam. Among the first 25 cold masses, $44 \%$ did not respect the tolerance concerning the local twist. The tolerance for the average twist was respected in all cases. The criterion used to limit the twist was therefore reviewed. The impact of the twist on the magnetic field of the dipole cold mass can be computed as

$$
I_{0}=\frac{1}{\langle B\rangle} \cdot \int_{0}^{L} B(y) \cdot[F D(y)-\langle F D\rangle] \cdot(L-y) \cdot d y
$$

where $F D(y)$ is the local field direction as a function of the longitudinal position, $\langle F D\rangle$ is the value of $F D(y)$ averaged on the cold mass length $(L), B(y)$ is the norm of the local magnetic field as a function of the longitudinal position and $\langle B\rangle$ is the average value of $B(y)$ along the magnet axis.

It was therefore decided to use the $\mathrm{I}_{0}$ criterion (with a tolerance equal to $\pm 0.18 \mathrm{rad} \cdot \mathrm{m}^{2}$ ) instead of the former criterion for the local twist, since this last was too restrictive and did not respond to a real need. Following this new criterion, all the first 25 produced cold masses were within the limitation of $\pm 0.18 \mathrm{rad} \cdot \mathrm{m}^{2}$. The mean of the resulting distribution was zero and the standard deviation equal to $0.05 \mathrm{rad} \cdot \mathrm{m}^{2}$. The reference value at $3 \sigma, 0.15 \mathrm{rad} \cdot \mathrm{m}^{2}$, was of the same order as the defined tolerance of $\pm 0.18 \mathrm{rad} \cdot \mathrm{m}^{2}$. Concerning the average tilt, all cold masses were within the tolerance with a mean of the distribution equal to $-0.016 \mathrm{mrad}$ and a standard deviation of $0.05 \mathrm{mrad}$. The value of $3 \sigma, 0.15 \mathrm{mrad}$, was much smaller than the tolerance of $\pm 1 \mathrm{mrad}$ for the average tilt [2].

\section{REVIEW OF THE SHAPE TOLERANCES}

\section{A. Bending the Dipole Cold Masses}

The dipole cold mass curvature in the transversal plane is obtained by constraining the magnet on a curved press bench and, under press load, welding the two half-cylinders to form a skin around the collared coils trough the magnetic yoke. When the load is released after welding, the magnet loses a nonnegligible fraction of its curvature, due to elastic spring back (aprox. 23\%). To compensate the spring back, the press bench is shaped to a slightly higher curvature (smaller radius) than that of the final desired curvature of the cold mass (See Fig. 2).

The half-cylinders are made of stainless steel grade AISI316LN. They are pre-bent with an initial nominal sagitta of $9 \mathrm{~mm}$, but in some cases up to $20 \mathrm{~mm}$ sagitta was measured. There is a concave and a convex half cylinder for each dipole cold mass. In some cases, after application of the above-described procedure of bending, the elastic spring back of the dipole cold mass is such that the shape of the dipole is not within

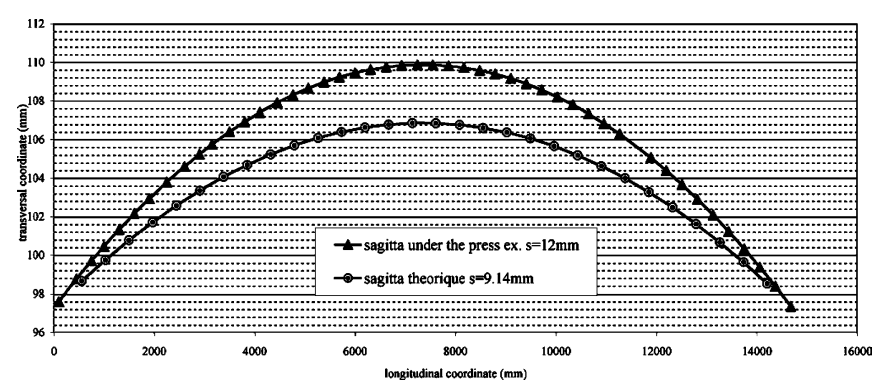

Fig. 2. The theoretical shape of the dipole cold mass at the following stages of the assembly: under the press (under load) and after the longitudinal welding (unloaded).

the required tolerance. In order to correct the shape, a special procedure was introduced. Each cold mass, needing correction after the longitudinal welding of the half cylinders, was placed on a "re-shaping bench" on two supporting points and deformed in several steps until the nominal shape is reached within the specified tolerances [1]. Other technique of correction has been tried by making a local heating of the cylinder with welding strips on the outside surface of the shrinking cylinder. Once the dipole cold mass assembly is finished, the correctors cannot be re-positioned without opening the dipole cold mass.

\section{B. Sagitta of the as-Built Dipoles}

In order to compare the different cold masses between them, although this would be not the acceptance criterion, we can calculate the real sagitta of each of the cold masses. The algorithm that is used to determine the sagitta, is based on the 3D coordinate transformation, known as Helmert transformation. The axes of the two cold bore tubes are analyzed simultaneously and the $\mathrm{z}$ (vertical) coordinates are neglected. Then the measured data are compared with a theoretical shape, discretized in the same number of points as measurements. Over a fixed cord-length (equivalent to the magnetic length of the cold mass), with different values of sagitta, different ideal shapes are calculated. Those shapes are compared by the $2 \mathrm{D}$ transformation with the measured data. By minimizing the errors between the ideal shapes and the measured values, after a number of iteration, the sagitta of the cold mass is found. For the calculations, the measurements done in the two beam channels are considered simultaneously and with the same weight [3]. Fig. 3 shows the sagitta of the cold masses after the longitudinal welding of the half cylinders. After the longitudinal welding, $63 \%$ of the dipoles were not within the required tolerance. Part of the shape error disappears during the final assembly by alignments of the end of the cold bore tubes, but still a significant percentage of them remains outside the base line tolerances, in the horizontal plane.

\section{Stability of the Shape}

The dipole cold mass constitutes a highly dissipative object, composed of a number of layers (coils, collars, yoke laminations, shrinking cylinder) contributing to the nonlinear response of the whole structure.

The main dissipative mechanism is based on the local stick-slip effect related to the Coulomb friction. The micro displacements occurring at the interfaces between the layers 


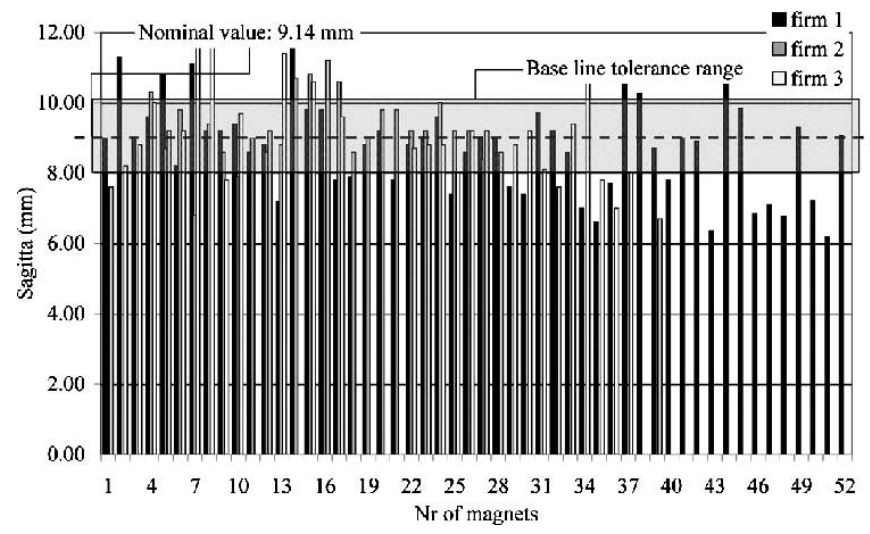

Fig. 3. The sagitta of the cold masses after longitudinal welding.

as well as between the collars and yoke laminations cause a dissipation of energy in the volume of the structure both under static and cyclic loads. As a result the structure shows a residual deformation and progressive evolution of curvature in the course of thermo-mechanical cycles. This phenomenon is similar to ratcheting in the elasto-plastic structures subjected to sustained and cyclic loads.

The activation of the dissipative mechanism depends on the way the magnets are assembled and varies between the manufacturers. Also, the initial curvature imposed to the magnets during the welding process, from which the magnets "spring back" to their "final" curvature differs between the manufactures (See Fig. 3).

In order to "shake" down the structure to the minimum of its potential energy it might be envisaged to excite the magnet (with longitudinal waves) so as to obtain an internal reconfiguration of the structure. Another possibility to "freeze" the magnet curvature is to block transversally the central foot inside the cryostat so as to obtain a kinematic control of the magnet shape. Finally, some other methods like the corrective welds might also be used, however, without guarantee on the stability in the long-term.

The geometrical instability of the cold mass has a direct effect on the position of the corrector magnets, which are placed inside the cold mass and fixed on the end plates. The corrective action was only applied on limited number of magnets and then stopped in the industry.

\section{Racetrack Tolerance, Ultimate Limit}

Due to the difficulties encountered, the requirements on the shape were reviewed. A new shape for the tolerance range and an ad hoc sorting of the magnets during installation were proposed (See Fig. 4). A cold mass having its shape outside of the new racetrack tolerance it would be installed in the middle of the cell (about $30 \%$ of the magnets can have this location) where the beam size is larger and allows a larger set of tolerances: the ultimate racetrack. In addition, these dipoles might be installed following a specified procedure of alignment by the ends, if the cold bore tube extremities could not be centered on the geometrical axes [4]. Outside the ultimate tolerance range, a magnet is rejected.

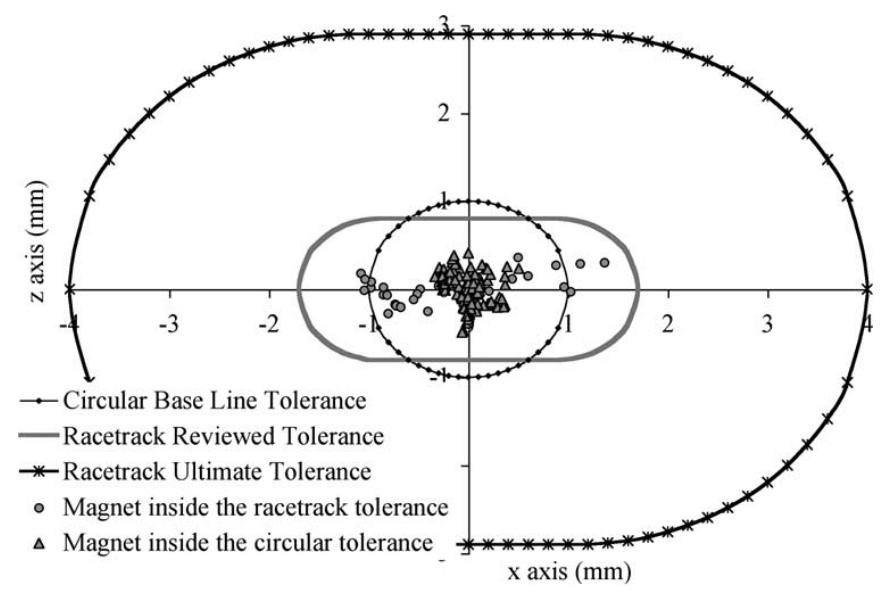

Fig. 4. Tolerances. Example of magnets with respect to the different tolerances.

\section{CONCLUSION}

The review of the tolerances has been carried out taking into account the industrial feasibility and the necessity for the good installation and functioning of the LHC, from geometry point of view. The new tolerances assure also a reduction of production time. Unacceptable geometrical instability of the dipoles was observed on magnets assembled with nonstandard procedures, which was mainly different from the standard one by the additional shape-correction procedure. The change of geometry of a dipole, after the final assembly, has a direct effect on the position of the corrector magnets, which cannot be corrected after the cold test. A new strategy of acceptance was proposed to suppress the unstable corrective actions. This new procedure is such that the magnets should be sorted during the installation in order to place the magnets with smaller mechanical aperture in the middle of the cells.

\section{ACKNOWLEDGMENT}

The authors would like to thank the members of the CERN Working Group on Alignment for the useful discussions and recommendations, to S. Fartouhk (CERN) for helping them to review the tolerances on the twist and on the mechanical aperture of the dipole cold masses.

\section{REFERENCES}

[1] M. Bajko, R. Chamizo, and A. Pardons, "Influence of geometrical parameters on the flexural rigidity of the LHC dipole cold mass assembly," IEEE Trans. Appl. Supercond., vol. 12, no. 1, pp. 1713-1717, March 2002.

[2] R. Chamizo, "Analysis of the Twist on the LHC Dipole Cold Masses,", Internal Note AT MAS CERN, February 2003.

[3] M. Bajko, Geometry of the Dipole Cold Masses. Results on the First 20 Cold Masses, Produced at Ansaldo, CERN AT MAS, October 2003.

[4] G. Gubello, M. L. China, and W. Scandale, "Summary of LHC Dipole Shape Produced Up to July 2003,", LHC Project Report 670, August 2003. 